\title{
Innovación curricular en la educación superior: Experiencias vividas por docentes en una Escuela de Enfermería
}

\author{
Curricular innovation in higher education: experiences lived by teachers in a school of nursing
}

\author{
Verónica Guerra-Guerrero1* orcid.org/0000-0003-4517-6678 \\ Carmen G. Miño-González¹ orcid.org/0000-0002-1903-4492 \\ Margarita Poblete-Troncoso' orcid.org/0000-0003-4068-1253 \\ Carmen G. Cofré-González¹ orcid.org/0000-0002-9318-5483 \\ Paula Ceballos-Vásquez ${ }^{1}$ orcid.org/0000-0002-3804-5146
}

Ana Jara-Rojas ${ }^{1}$ orcid.org/0000-0003-4439-9714

1 Universidad Católica del Maule. Talca, Chile

Fecha de recepción: Abril 12 - $2017 \quad$ Fecha de revisión: Diciembre 4 - $2017 \quad$ Fecha de aceptación: Diciembre 21 - 2017

Guerra-Guerrero V, Miño-González CG, Poblete-Troncoso M, Cofré-González CG, Ceballos-Vásquez P, Jara-Rojas A. Innovación curricular en la educación superior: experiencias vividas por docentes en una Escuela de Enfermería. Univ. Salud. 2018;20(1):53-63. DOI: http://dx.doi.org/10.22267/rus.182001.109

\section{Resumen}

Introducción: En las últimas décadas, en Chile, la formación profesional en enfermería ha sufrido transformaciones en sus planes de estudio, pasando de tener un currículo tradicional a uno basado en competencias, en concordancia con las actuales exigencias del mercado laboral. Objetivo: Analizar dos temáticas derivadas del estudio centrado en comprender las experiencias de vida de docentes de la Escuela de Enfermería de la Universidad Católica del Maule en Chile, sobre la innovación curricular. Materiales y métodos: Estudio fenomenológico, mediante entrevistas en profundidad, a veinte docentes que han participado en la formación de enfermeros. Muestreo por conveniencia y de acuerdo al criterio de saturación de los datos. El análisis de datos fue de tipo temático según lo propuesto por Van Manen. La investigación fue aprobada por el Comité de Ética de la institución. Resultados: Se identificó siete temas principales, dos de los cuales son analizados en este artículo: la innovación como un proceso complejo y los cambios sin cambios. Conclusiones: La transición de un currículo tradicional hacia uno basado en competencias, desde la experiencia de los docentes, implica desafíos complejos y profundos cambios, frente a los cuales, las instituciones de educación superior tienen que proporcionar las condiciones para facilitar dicho proceso.

Palabras clave: Educación superior; educación en enfermería; currículum; educación basada en competencias; educación profesional. (Fuente: DeCS, Bireme).

\begin{abstract}
Introduction: In recent decades, in Chile, vocational training in nursing has undergone transformations in their study plans, moving from having a traditional curriculum to one based on competencies, in accordance with the current demands of the labor market. Objective: To analyze two topics derived from the study focused on understanding the life experiences of teachers in a Nursing School on the curricular innovation. Materials and methods: A phenomenological study through in-depth interviews was conducted to twenty teachers, who have participated in the training of nurses. Sampling for convenience was done according to the data saturation criterion. The data analysis was thematic type as proposed by Van Manen. The research was approved by the Ethics Committee of the institution. Results: Seven main themes were identified, two of which are analyzed in this article: innovation as a complex process and unchanged changes. Conclusions: The transition from a traditional curriculum to one based on competences,
\end{abstract}


from the experience of teachers, implies complex challenges and profound changes; considering this, higher education institutions have to provide the conditions to facilitate such a process.

Keywords: Education, higher; education, nursing; curriculum; competency-based education; education, professional. (Source: DeCS, Bireme).

\section{Introducción}

Con el surgimiento de la globalización, la educación superior inició cambios trascendentales en la formación profesional debido fundamentalmente a las exigencias del entorno laboral(1). Tal como fue declarado por la Organización de las Naciones Unidas para la Educación la Ciencia y la Cultura (UNESCO)(2), la formación de profesionales competentes y comprometidos con el desarrollo social constituye hoy en día una misión esencial de la educación superior.

La sociedad actual demanda la formación de profesionales capaces no sólo de resolver con eficiencia los problemas de la práctica profesional, sino que también puedan lograr un desempeño profesional ético, con responsabilidad ciudadana, con compromiso social y con valores asociados al desempeño profesional. El desafío de formar profesionales con estas características implica el desarrollo de competencias que deben ser adquiridas mediante un currículo que propenda la integración de $\operatorname{estas}^{(3)}$.

En este sentido, es posible señalar que los currículos basados en competencias tienen su génesis en la Declaración de Bolonia el año 1999, en la que se sugirió que la enseñanza superior debía ser coherente, compatible y competitiva, que permita titulaciones reconocibles $y$ comparables, que favorezcan la movilidad profesional, particularmente en Europa. Actualmente, la mayoría de las instituciones de educación superior se encuentran innovando su educación y evaluando las prácticas, en las cuales las competencias son vistas como un todo integrado de conocimientos, habilidades y actitudes $^{(4)}$.

En la disciplina de enfermería, los cambios en la educación superior comenzaron a surgir a partir del año 2001, también en Europa, a través del Proyecto Tuning Educational Structures in Europe II (Tuning) ${ }^{(5,6)}$ que convocó a trece países de la Comunidad Económica Europea para desarrollar, entre otras actividades: perfiles profesionales basados en competencias, establecer resultados de aprendizaje, intercambiar información curricular, de modo que pueda crear una base de comparabilidad y transparencia, y facilitar la movilidad y convergencia en la educación superior europea(6). Todo lo anterior como una forma de resguardar y mejorar la calidad de la educación.

Por su parte, la Organización Mundial de la Salud(7), en el año 2011 y 2009 publicó dos informes de educación en enfermería con el objetivo de definir las competencias requeridas y los estándares generales para la educación inicial de las enfermeras. Del mismo modo, el Consejo Internacional de Enfermeras ha destacado la importancia de continuar desarrollando las competencias en enfermería como una responsabilidad profesional(8).

Dentro de los procesos de transformación e innovación que las instituciones de educación superior han desarrollado en los últimos años, se encuentra la producción de diseños curriculares orientados hacia el desarrollo de competencias profesionales ${ }^{(9)}$. Un perfil de egreso estructurado sobre la base de competencias permite dar cuenta de las habilidades que tienen los estudiantes al finalizar sus estudios, asegurando de este modo una mejor integración al mundo de las organizaciones en el futuro, por cuanto, estos pueden evidenciar en sus desempeños el valor que agregan como profesionales, siendo la institución formadora la responsable y garante de tales competencias(3,10,11). El diseño curricular basado en competencias es un documento elaborado a partir de la descripción del perfil profesional, en el que se relacionan los desempeños esperados de una persona en un área 
ocupacional, para resolver los problemas propios de su ejercicio profesional(1).

El concepto de competencias, ha sido definido de diversas maneras, sin embargo, una descripción común indica que la competencia consiste en varias partes conectadas de conocimiento, habilidades, actitudes y valores que pueden ser usadas para resolver adecuadamente un problema(7,11).

Por su parte, la innovación curricular que lleva a la transición de un currículo tradicional a uno por competencias, incluye una serie de cambios por parte de las instituciones y sus actores. Estos cambios involucran desafíos que las instituciones deben superar para implementar el currículo basado en competencias; los desafíos han sido clasificados por autores como Schmal(12) en tres aspectos: el diseño curricular y la docencia, la gestión institucional y el sistema global.

En la disciplina de enfermería a nivel internacional y particularmente en Chile, también se comenzó a realizar cambios en la formación profesional para dar respuesta a las necesidades actuales de salud de la población y según la realidad actual que exige capacidad de adaptación, flexibilidad, e interés en la actualización. En las últimas décadas la educación en enfermería ha tenido que centrar la formación en profesionales que den cuenta de un recurso humano capacitado, con saberes actualizados, pero por sobre todo competente(6).

Aunque a nivel internacional se ha descrito que las competencias de enfermería en salud pública fueron incorporadas en el año 1931, en Chile, la mayoría de los actuales currículos y perfiles profesionales de enfermería tan sólo en esta última década comenzaron con el proceso de cambio e innovación, encontrándose que existen modificaciones en el proceso de enseñanzaaprendizaje, pasando de un currículo tradicional o convencional (centrado en el profesor), a uno innovado (centrado en el estudiante)(6) basado en competencias. El currículo tradicional se centra en la acumulación de conocimientos, los protagonistas son los profesores, quienes proporcionan los contenidos y los estudiantes quienes reciben éstos, asumiendo un rol pasivo y limitado ${ }^{(1,11)}$. Por el contrario, en el currículo basado en competencias, el estudiante es quien asume un rol protagónico y los conocimientos se adecuan a la realidad social y productiva del medio laboral.

La Escuela de Enfermería de la Universidad Católica del Maule en Chile, se adhirió a la formación mediante un currículo basado en competencias, en el año 2013, de acuerdo a los lineamientos de la institución y como parte de un proyecto conjunto con otras Escuelas de Enfermería del país. Este proceso de innovación educativa permitió realizar cambios de acuerdo a las necesidades actuales, requiriendo evaluación permanente y rigurosa con incorporación de todos los involucrados: estudiantes, docentes, administrativos y directivos, de esta manera realizar ajustes para lograr una formación profesional de calidad.

En la actualidad, la Escuela de Enfermería se encuentra en el tercer año de innovación curricular y se desconoce la experiencia que ha significado este proceso tanto para estudiantes como para docentes. Por otro lado, considerando que la transición desde un currículo tradicional a otro innovado, requiere adaptación y genera crisis entre los participantes, resulta relevante desarrollar estudios que permitan conocer las experiencias que presentan los involucrados. En este sentido, se ha planteado que realizar la evaluación de la innovación curricular es una actividad que se debería desarrollar desde que se inicia el cambio en la malla(11), es así como se describe que en países como Estados Unidos, esta evaluación comienza en fases iniciales(13).

Este artículo es parte de la investigación desarrollada con el objetivo de comprender las experiencias vividas por estudiantes y docentes de la Escuela de Enfermería, acerca del cambio en la formación, desde un currículo tradicional a uno basado en competencias. Por lo tanto, tiene como objetivo presentar dos temáticas que derivaron del análisis realizado a las experiencias vividas por los docentes, con respecto a la innovación curricular. Dichos temas son: "la innovación como un proceso complejo" y "los cambios sin cambios". 


\section{Materiales y métodos}

Estudio cualitativo con enfoque fenomenológico hermenéutico, basado en Van Manen que buscó comprender las experiencias de vida de docentes sobre los desafíos que involucra la innovación curricular. La fenomenología hermenéutica según Van Manen es una metodología descriptiva (fenomenología), debido a que está centrado en comprender como las cosas aparecen, es decir, deja que las cosas hablen por sí solas; y también es una metodología interpretativa (hermenéutica) porque afirma que no hay cosas que no puedan ser interpretadas ${ }^{(14)}$.

Se realizó un muestreo por conveniencia a los docentes que participan en la formación académica de estudiantes de la carrera de enfermería en una Facultad de Ciencias de la Salud de la Universidad Católica del Maule (Chile). Todos los participantes incluidos en el estudio habían experimentado el proceso de la innovación curricular(15).

Los criterios de inclusión para la selección de los docentes fueron los siguientes: 1) que pertenecieran a la Escuela de Enfermería; 2) que colaboraran en el proceso formativo de los estudiantes y que no pertenecieran a la Escuela de Enfermería; y 3) aquellos que voluntariamente estuvieran de acuerdo con participar y que firmaron el consentimiento informado. El muestreo se definió según la disponibilidad de los participantes y basado en el criterio de saturación de los datos(15).

Los datos fueron recolectados mediante entrevistas en profundidad, notas de campo y observación participante(15). Las entrevistas fueron realizadas individualmente, basadas en preguntas con respuestas abiertas, generales y centradas en comprender el fenómeno principal del estudio. En este tipo de entrevistas el entrevistador tiene la facultad de introducir preguntas adicionales para precisar conceptos $\mathrm{u}$ obtener mayor información sobre los temas esenciales y permite generar un ambiente de conversación, tal como lo plantea Van Manen(14), asimismo, Creswell(15) afirma que es una forma de validar las preguntas de la entrevista.
Se utilizó preguntas abiertas, envolventes y no direccionales, centradas en lograr el objetivo general de la investigación, por esto se utilizó preguntas clave tales como ¿qué? o ¿cómo?, más que, ¿por qué? como una forma de explorar el fenómeno central de esta investigación. De este modo, el protocolo de preguntas que guió la entrevista se basó en una pregunta inicial que dio respuesta al objetivo general de esta investigación (pregunta 1); seguido por subpreguntas (pregunta 2 y 3), que respondieron a los objetivos específicos de ésta. Además, se incorporó durante la conversación otras preguntas adicionales, relacionadas con el fenómeno de estudio ${ }^{14}$. Las preguntas que guiaron las entrevistas fueron las siguientes:

1. ¿Cuál es su experiencia vivida con el proceso de innovación curricular en la carrera de enfermería?;

2. ¿Cuál es su experiencia vivida con respecto al proceso formativo enfrentado por los estudiantes que se encuentran en innovación curricular?; y

3. ¿Cuál es su experiencia vivida frente al cambio desde un currículo tradicional a uno basado en competencias?

Cada una de las entrevistas se llevó a cabo en una fecha acordada según la disponibilidad de los participantes. Se efectuaron en las salas de reuniones y oficinas de los participantes, ambientes que proporcionaron la privacidad y acústica adecuadas para realizar correctamente los registros a través de una grabadora digital de alta fidelidad, desde la cual el audio fue transferido a un ordenador, posteriormente, fueron transcritas palabra por palabra por la investigadora principal. Tanto el audio como el registro escrito de las entrevistas se mantuvieron resguardadas para respetar la confidencialidad de los sujetos de estudio, además, se le otorgó un nombre ficticio a cada participante.

El análisis de datos se realizó de forma manual, basados en Van Manen quien propone el análisis temático de los datos siguiendo tres enfoques: 1 ) holístico; 2) selectivo; y 3) detallado (14). El análisis permitió identificar siete temas principales, dos de los cuales se presentan en este artículo. Para 
cumplir con la rigurosidad científica en investigación se consideró las estrategias de validación y confiabilidad propuestas por Creswell(15).

\section{Consideraciones éticas}

Antes de recolectar los datos, esta investigación fue sometida a evaluación por el Comité de Ética Científico de la Universidad Católica del Maule, en donde fue aprobada para su ejecución. Adicionalmente, se solicitó autorización a las respectivas autoridades administrativas de la Escuela de Enfermería quienes permitieron la realización de la investigación. Todos los docentes que estuvieron de acuerdo con participar en el estudio firmaron el consentimiento informado respectivo.

\section{Resultados}

Se realizó un total de 20 entrevistas en profundidad a académicos que participan en la formación de los estudiantes de la Escuela de Enfermería de una institución de educación superior. La duración de las entrevistas fue de 13 a 50 minutos.

Respecto a las características de los participantes (Tabla 1), se encontró que los entrevistados tenían entre 33 y 65 años de edad, con un promedio de 45 años; la mayoría era de sexo femenino; llevaban trabajando en la institución entre 3 y 27 años, con un promedio de 11 años; la mayoría de ellos tenía contrato indefinido; y sólo uno de ellos trabajaba además, en otra institución de educación.

Tabla 1. Características generales de los docentes participantes

\begin{tabular}{ll}
\hline \multicolumn{2}{c}{ Características generales } \\
\hline Edad & 33 a 65 años \\
Sexo & 4 hombres \\
& 16 mujeres \\
Antigüedad en la institución & 3 a 27 años \\
Situación contractual & 19 contrato indefinido \\
& 1 honorarios \\
Trabaja en otra institución & 1 participante \\
\hline
\end{tabular}

Los docentes que participaron en el estudio pertenecían al Departamento de Enfermería, y también al Departamento de Ciencias Básicas, encontrándose no sólo profesionales de enfermería, sino también biólogos, químicos, antropólogos, sociólogos, entre otros.

Este artículo aborda dos temáticas derivadas del análisis que corresponden al tema "La innovación curricular es un proceso complejo" y a "Los cambios sin cambios" (Tabla 2). Otros temas identificados, que no son incluidos en este artículo fueron: "Innovación, una experiencia enriquecedora"; "El conocimiento para el conocimiento"; "Experiencia con estudiantes"; "Las dificultades"; y "Lo pendiente".

Tabla 2. Descripción de temas y subtemas derivados del análisis de entrevistas

\begin{tabular}{ll}
\hline \multicolumn{1}{c}{ Temas } & \multicolumn{1}{c}{ Subtemas } \\
\hline Tema 1: & Sobrecarga laboral \\
"La innovación es & Tiempo \\
un proceso & Proceso de transición \\
complejo" & Proceso abrupto y apurado \\
Tema 2: & Cambios en metodologías \\
"Cambios sin & educativas \\
cambios" & Cambios en proceso de \\
& enseñanza-aprendizaje \\
& Cambios en el sistema de \\
& evaluación \\
& Transición sin cambios \\
& Formación sin cambios \\
& Repetición de malla antigua \\
\hline
\end{tabular}

\section{Tema 1: "La innovación curricular es un proceso complejo"}

Los participantes describieron que la innovación ha involucrado aspectos complejos que se encuentran en transición y que por lo tanto no ha finalizado. Esta complejidad fue descrita en términos de la rapidez con que fue implementada la innovación curricular y que no consideró un período de marcha blanca. También señalaron que han experimentado este proceso en forma compleja, porque durante el proceso continuaron trabajando el mismo número de personas, no obstante que ocurrió un nuevo proceso. Es así como uno de los participantes señaló lo siguiente: 
“...de repente como que nosotros mismos nos hemos sobrecargado, en nuestra carga laboral, y eso mismo nos ha pasado la cuenta, yo encuentro que ha sido un proceso largo, complejo y cansador, no ha sido fácil, porque tenemos toda esta malla antigua que se está terminando, tenemos malla nueva y estamos trabajando en algo nuevo, entonces estamos con lo mismo de antes, y que tenemos que ejecutarlo". Entrevista \#1; línea 3340.

Asimismo, describieron la dualidad ocurrida respecto a las mallas curriculares $y$ principalmente por la transición que surgió con la implementación de una nueva malla curricular, como lo expresó uno de los participantes:

"Claramente es una mayor carga el hecho, no de la innovación en sí, sino el hecho de estar con mallas paralelas, como es una Escuela nueva estábamos preparando clases de malla antigua y malla nueva, en paralelo, en muchas ocasiones, y eso fue, eso ha sido un poco más complejo, ha sido difícil, de compatibilizar". Entrevista \#2; líneas 106-111.

Con respecto al tiempo, uno de los participantes señaló lo siguiente:

"Si, yo creo que deberíamos habernos dado tiempo, horario, donde se trabajaran las áreas antes de trabajar juntos, pero se destinara tiempo, porque siempre hoy día, pero es que hoy día yo tengo reunión, tengo clases, entonces, coordinar eso, es muy difícil, entonces yo creo que debería haberse exigido a los docentes por las áreas que corresponden, haber tenido horas destinadas a trabajo conjunto, previo, con una buena información, con una buena formación, y como te digo, para que tú te convenzas de lo que tú estás haciendo, es lo correcto, ya como que partimos y nos lanzamos...., pero es una cuestión muy compleja, muy compleja". Entrevista \#4; líneas 103-116.

\section{Tema 2: "Los cambios sin cambios"}

Los participantes describieron sus experiencias con la innovación curricular, desde una doble perspectiva, existiendo experiencias relacionadas con un proceso de cambios y otro sin cambios. El proceso de cambios fue descrito en relación a las metodologías educativas, al proceso de enseñanza-aprendizaje y el sistema de evaluación. Por su parte, el proceso sin cambios fue atribuido a la transición entre el currículo tradicional y el innovado (basado en competencias), en la formación y en la malla antigua que son vistos por los docentes como procesos que continúan siendo iguales que antes de la innovación.

Respecto a los cambios, los participantes manifestaron que el proceso de la innovación curricular generó cambios importantes en las metodologías educativas. En este sentido, uno de los participantes manifestó que:

"Nosotros lo vemos ahora, con los de cuarto, porque ya llevamos cuatro años, y es agradable, hoy día tuvimos reunión y se veían estudiantes más maduros, que entendían lo que se les hablaba en relación a, hay mucho acercamiento, a las metodologías que tienen relación con autoaprendizaje, y con una muy buena recepción porque al principio cuando eran estos mismos estudiantes de primero, tú le hablabas de estos conceptos y ellos como que, como que no entendían de lo que uno les hablaba, en cambio ahora ellos entienden, hay un concepto más de autonomía, o sea, se logró la autonomía que se buscaba, y a mí me parece positivo, que es lo que buscamos". Entrevista \#10; línea 363-378.

Las experiencias en relación con los cambios ocurridos con la innovación curricular fueron vinculadas principalmente con los estudiantes y en cómo ellos vivieron esos cambios; en este sentido, uno de los participantes describió lo siguiente:

"Yo creo que los chicos han podido ver como es la diferencia entre la formación, me refiero a, a los que han estado en la malla por objetivos, yo puedo comparar, esta malla por competencias, con los nuevos ingresos que han ido habiendo...., los estudiantes están viendo este abordaje que es mucho más integral, versus el que quizás nosotros tenemos, que es tal vez un poquito más cuadrado, de ver, es como un poco, un poco más rígido, y como que hay algunas cosas que incluso a solicitud de ellos mismos hemos ido incorporando, talleres o 
actividades que están en la malla de competencias". Entrevista \#7; líneas 152-165.

Mediante sus experiencias de vida, los participantes que describieron la innovación curricular como un proceso sin cambios, indicaron que la formación de los estudiantes actualmente no presenta diferencias en relación a la malla antigua, la cual tiene un enfoque tradicional. Señalaron, además, que en la formación de los estudiantes, en algunos casos, se produce la repetición de contenidos, lo cual no evidencia un cambio en la malla curricular.

En concordancia con aquellos participantes que describieron la innovación curricular como un proceso de "sin cambios", uno de ellos señaló lo siguiente:

“...el currículum lo estamos haciendo por competencias, pero en la mirada global de ciertos módulos, finalmente sigue siendo igual por objetivos" Entrevista \#7; líneas 289-221.

Del mismo modo, el participante señaló lo siguiente:

"...siento que el abordaje que se dio de ciertos módulos no dice relación con la formación de competencias, sino que es una forma tal vez un poco más maquillada de la misma formación que estábamos haciendo por objetivos..." Entrevista\#7; líneas 304-308.

El proceso sin cambios fue también descrito por otros participantes de la siguiente forma:

"En esencia, el curso mío, yo diría que esencialmente no ha cambiado, tengo la misma cantidad de módulos....así que yo, diría que esencialmente, en lo que es mí tópico no he visto mayor diferencia, no, no se me ha pedido tampoco, porque yo envíe, por ejemplo, cuando hicimos los programas, eran esencialmente la misma materia, sí bueno, es que es difícil que la materia va a cambiar en realidad, pero el enfoque, es como más o menos lo mismo, que ellos tienen laboratorio y las clases teóricas" Entrevista \#9; líneas 42-45; 6167.

\section{Discusión}

En relación con los hallazgos de esta investigación, es posible señalar que las características de los docentes que participaron en este estudio coinciden con lo encontrado en otro estudio de tipo cuantitativo realizado también en Chile(13), en donde la mayoría de ellos fueron mujeres, con experiencia laboral promedio de 11 años en el área de educación. Los resultados de la investigación muestran que el perfil que presentan los docentes que trabajan en educación superior, en el área de salud, es similar en las casas de estudios del país y que se encuentran también desarrollando la innovación curricular basada en competencias.

Acerca del primer tema descrito en los resultados de esta investigación, es posible señalar que se evidencia que los docentes experimentan la innovación como un proceso complejo que involucra tiempo y una mayor carga laboral, esta carga tiene que ver con el cumplimiento que tienen que dar al desarrollo de dos mallas paralelas, una malla tradicional y una innovada, similar a lo descrito en otro estudio realizado en el contexto chileno(13).

La complejidad del proceso, junto con el cambio que se produce en la formación de los estudiantes, al incorporar un currículo basado en competencias, fue descrita por los participantes en términos de desafíos que se producen principalmente en las metodologías de enseñanza. Esto es lo que según algunos autores, debe ser atendido por las instituciones de educación superior, implicando para ello gestionar profundos cambios en la forma de enseñar, puesto que la transición de un currículo tradicional a uno basado en competencias no es fácil e implica movimiento en las estructuras de la institución que realiza el proceso de cambio(1).

Estas complejidades, que fueron manifestadas por los docentes, también se plantean en otros estudios, en donde se señala que ocurren y se evidencian una vez que se ha implementado el nuevo currículo, cuando realmente se empieza a modificar este y por lo tanto, cuando se cambia la cultura de la institución(1). En este sentido, 
algunos autores sostienen que el logro de competencias individuales involucra enfoques que van más allá de lo convencional, y es aquí en donde surge la preocupación sobre el vacío de consenso y principalmente de la forma en cómo se definen, desarrollan, implementan y evalúan las competencias(16,17). Es por esto que a nivel internacional todavía se describe que existe heterogeneidad sobre las competencias que se requieren en la formación $(5,16,18)$.

Lo anterior probablemente es uno de los aspectos que generan la confusión declarada por los docentes de esta investigación, en términos de la complejidad que involucra la innovación curricular(18), ya que como se ha descrito, existen inconsistencias acerca de aspectos como el marco de referencia que se utiliza para definir las competencias, así como la carencia de enfoques y lenguaje estandarizado, constituyeron desafíos que afectan la adopción o transformación curricular(16). Entre las estrategias que se han reconocido en enfermería, para la integración e implementación de competencias, se encuentra el liderazgo que existe a nivel de los programas de estudio; este debe valorar y estar de acuerdo con los objetivos que tiene la implementación de competencias en el currículum; al respecto, alguno autores sugieren la utilización de modelos que guíen el proceso de la implementación del currículum y la ayuda hacia los educadores para que prioricen y planifiquen los pasos a seguir en su desarrollo(17).

Para los docentes que participaron en esta investigación, la innovación curricular se constituye como un proceso complejo, debido a que el paso desde un currículo tradicional a uno innovado fue llevado a cabo en forma abrupta y acelerada. Con base a la descripción de algunos autores, podría plantearse entonces que la incorporación acelerada se debe a que la innovación que se introduce en el currículum es una condición impuesta o que proviene sólo desde expertos o diseñadores curriculares, en donde no existe participación sustancial por parte de los docentes, sino que se produce una implantación unidireccional e inesperada(5,18). Contrario a esto, existe evidencia que muestra que cuando el currículum está conectado con las necesidades de los docentes, ellos se involucran no sólo profesionalmente, sino que también emocionalmente con el proceso de innovación ${ }^{(19)}$ y por lo tanto, esto podría reducir la complejidad con que se vive su implementación.

Esto concuerda con lo propuesto por González(1), al indicar que es necesario que cada docente asuma una perspectiva reflexiva sobre el cambio curricular y sobre las competencias, por lo que asumir el cambio demanda un trabajo conjunto, no sólo de los docentes, sino que también de la institución y comunidad universitaria.

Respecto al proceso de enseñanza aprendizaje, en esta investigación se evidenció que la innovación curricular ha llevado a que los docentes modifiquen las metodologías de enseñanza, guiando a los estudiantes hacia el desarrollo de un trabajo autónomo, coincidiendo con los objetivos iniciales del Proyecto Tunning II desarrollado en Europa ${ }^{(5,6)}$, ya que la educación por competencias se basa en la corriente constructivista del aprendizaje, que considera al estudiante como portador de conocimiento, el cual resulta de su interacción con el ambiente o contexto(18-20). Este trabajo autónomo se acompaña de mayor calidad en el aprendizaje alcanzado por los estudiantes, observándose también, por parte de los docentes, que existe una mayor autonomía y motivación por aprender en la toma de decisiones basado en el conocimiento ${ }^{(21)}$. Este último, es reconocido como un elemento esencial para desarrollar las competencias, junto a las habilidades y actitudes que se requieren en el ejercicio del profesional de enfermería(22).

Para lograr lo anterior, es primordial que las instituciones de educación superior asuman como estrategia, la formación de los profesores, capacitándolos en procesos metodológicas efectivos, para que los apliquen y logren los resultados que se espera en el currículum (18). De ese modo, reducir también la complejidad que declaran los docentes acerca de la innovación como proceso continuo y duradero en el tiempo, que requiere de apoyo hacia los profesores principalmente desde la institución formadora(19). Por su parte, respecto al segundo tema "Cambios sin cambios", es posible señalar que la autonomía 
de los estudiantes es valorada por algunos docentes de esta investigación, como un cambio que marca la innovación curricular y que diferencia el currículo tradicional del que se realiza basado en competencias, esto coincide con lo propuesto por Schoneman et al.(22), quienes plantean que la implementación del currículum basado en competencias se recomienda tanto a nivel de pre como posgrado. El cambio de roles que surge con la innovación, genera en los estudiantes una posición en la que el conocimiento se origina también a partir de ellos, lo cual coincide con otros estudios ${ }^{(1,13)}$.

Sin embargo, este cambio o transformación fue descrita también por algunos docentes como un proceso de progreso sin cambios, principalmente en la forma de entregar los contenidos teóricos. Esto es similar a lo descrito por Díaz(18), quien señala que los profesores trabajan, supuestamente, bajo una nueva estructura curricular, pero que en el fondo mantienen las mismas prácticas educativas que tenían en el currículum anterior. En ese sentido, González ${ }^{(1)}$ plantea que para que ocurra el cambio, es necesario tener un conocimiento acabado del trabajo desarrollado por docentes y estudiantes en su interacción con el conocimiento; considerar además, que las prácticas de enseñanza utilizadas en el currículo tradicional sean efectivamente renovadas a través de la innovación curricular, con el fin de seguir con nuevas formas de enseñar y aprender, para lograr las competencias profesionales y no la memorización de conocimientos que en algunos casos son descontextualizados(1).

Para que ocurra el verdadero cambio en el currículo González(1) señala que uno de los primeros cambios debe ser que el docente se haga a un lado y permita que en el centro del proceso educativo se ubiquen los estudiantes y los procesos de aprendizaje, es por esto que se debe promover en los docentes la reflexión permanente acerca del cambio.

Otro de los elementos que podrían evidenciar cambios efectivos en la innovación curricular, lo constituye la evaluación de las competencias adquiridas por los estudiantes, lo cual se describe como una tarea compleja, que requiere de un sistema que permita evaluar los conocimientos necesarios en el actuar profesional, las habilidades o destrezas propias de la disciplina y las actitudes inherentes al profesionalismo(20). Además, se describe que hay ausencia de métodos que efectivamente evalúen el logro de las competencias alcanzado por los estudiantes(17).

Finalmente, el hecho de que algunos docentes describieran que el cambio curricular no estaba ocurriendo, probablemente se debe a que la mayoría de los cambios siempre encuentran alguna resistencia ${ }^{(1,18)}$, por parte de quienes tienen que hacerlo, tal como lo indican algunos expertos en gestión de cambio(1). Se describe que se cuestiona escasamente acerca de cómo aprenden los profesores, qué los motiva a cambiar o no sus prácticas educativas, qué proceso es el que ocurre cuando existe la innovación o cuáles son las condiciones que deben darse para que en efecto ocurra el cambio o transformación curricular(18).

La resistencia al cambio tendría varias razones, entre las que se encuentran las creencias de que el cambio es negativo, que altera la relación formal, psicológica y social que el trabajador tiene con la institución; requiere que los docentes abandonen ciertos hábitos; que la institución no proporciona información suficiente sobre las razones del cambio, los beneficios que tiene el cambio o como irá progresando ese cambio; o que la estructura organizacional tradicional, la tecnología, no están alineados con el cambio(1).

Considerando que los docentes constituyen parte fundamental de los cambios curriculares, se hace necesario entonces, tener presente que el rol que debe asumir el docente en el proceso de innovación curricular tiene que ser activo, puesto que se considera como un referente y especialista en la formación profesional(18). También se debe incluir en la incorporación del proyecto curricular a los estudiantes, especialistas curriculares y disciplinares, representantes de la comunidad y particularmente a las instituciones de educación superior. 


\section{Conclusiones}

El cambio de un currículo tradicional, hacia uno basado en competencias, desde la experiencia de los docentes, implica desafíos complejos y un proceso de profundos cambios, frente a los cuales, las instituciones de educación superior tienen la responsabilidad de proporcionar las condiciones para facilitar dicho proceso.

Frente a esto, se requiere un liderazgo en el que los docentes sean considerados, no solo para la implementación de las innovaciones curriculares, sino que también desde el inicio, cuando se gesta $\mathrm{y}$ formula el proyecto de innovación.

Respecto a la complejidad que involucra el proceso de innovación, es fundamental que exista un trabajo colaborativo, en el que los docentes se sientan apoyados y reciban acompañamiento permanente, por parte de la institución educativa, durante todo el desarrollo del currículo. Junto a esto, es necesario homogenizar y/o adoptar un marco de trabajo que sea conocido y reconocido por todos los docentes involucrados, a fin de lograr el compromiso real que se necesita para el cambio. Para esto, es fundamental que los docentes reciban capacitación o formación que les permita innovar verdaderamente, para que de ese modo no vivan el proceso como un cambio sin cambios efectivos. Es aquí en donde la institución educativa tiene el deber de brindar oportunidades de aprendizaje y también valorar la trayectoria y experiencias que tienen los docentes.

\section{Recomendaciones}

Con base a los resultados de esta investigación es posible sostener que los docentes tienen un rol fundamental para lograr los cambios que necesita el sistema educativo por lo tanto se recomienda propiciar, por parte de las instituciones de educación superior, las condiciones que les permita a ellos realizar los cambios que necesita el actual sistema de educación.

\section{Agradecimientos}

Esta investigación fue posible desarrollarla gracias al financiamiento recibido por el Proyecto
Interno $\mathrm{N}^{\circ} 434176$ de la Universidad Católica del Maule.

\section{Conflicto de intereses}

Los autores declaran no tener conflicto de intereses.

\section{Referencias}

1. González Bernal MI, Sabana UdL. Currículo basado en competencias: una experiencia en educación universitaria. Educación y Educadores. 2006;9(2):95117.

2. UNESCO. Conferencia Mundial sobre Educación Superior. La Educación Superior en el Siglo XXI. Visión y Acción Paris: Unesco; 2006 [Available from: http://unesdoc.unesco.org/images/0011/001163/116 345s.pdf.

3. CINDA. Diseño Curricular Basado en Competencias y Aseguramiento en la Calidad de la Educación Superior Chile 2008 [Available from: https://www.cinda.cl/download/libros/39.pdf

4. Baartman LKJ, Bastiaens TJ, Kirschner PA, van der Vleuten CPM. Evaluating assessment quality in competence-based education: A qualitative comparison of two frameworks. Educational Research Review. 2007;2(2):114-29.

5. Moreno Olivos T. Competencias en educación. Una mirada crítica. Revista mexicana de investigación educativa. 2010;15:289-97.

6. Vásquez Aqueveque AM, Apablaza Correa R, Osorio Olivares L, Zuñiga Aguirre J. Construcción en red de un currículo basado en competencias. Ciencia y enfermería. 2011;17:35-42.

7. Blažun H, Kokol P, Vošner J. Survey on specific nursing competences: Students' perceptions. Nurse Education in Practice. 2015;15(5):359-65.

8. Orellana Y A, Sanhueza A O. Competencia en investigación en enfermería. Ciencia y enfermería. 2011;17:9-17.

9. CINDA. Evaluación del Aprendizaje en Innovaciones Curriculares de la Educación Superior Chile 2014 [Available from: https://www.cinda.cl/download/libros/2014\%20\%20Evaluaci\%C3\%B3n\%20de\%20los\%20aprendizaje s.pdf

10. Villardón Gallego ML. Evaluación del aprendizaje para promover el desarrollo de competencias. Educatio Siglo XXI. 2006;24:20.

11. Baartman LK, Prins FJ, Kirschner PA, van der Vleuten CP. Self-evaluation of assessment programs: a cross-case analysis. Eval Program Plann. 2011;34(3):206-16

12. Schmal S R, Ruiz-Tagle A A. Una metodología para el diseño de un currículo orientado a las competencias. Ingeniare Revista chilena de ingeniería. 2008;16:14758.

13. Rivas R E, Bustos M L, Rivas L Á, Lagos P X, San Martín G S. Experiencia subjetiva de estudiantes y docentes que 
vivencian un cambio curricular. Ciencia y enfermería. 2012;18:101-10.

14. Van Manen M. Researching lived experience. . 2da ed ed. Ontario, Canada: The Althouse Press; 1997.

15. Creswell J. Qualitative Iquiry and Research Design, Choosing Among Five Approaches. 3ra ed. ed. California, Estados Unidos.: SAGE Publications.; 2013.

16. Hawkins RE, Welcher CM, Holmboe ES, Kirk LM, Norcini JJ, Simons KB, et al. Implementation of competencybased medical education: are we addressing the concerns and challenges? Med Educ. 2015;49(11):1086102.

17. Dolansky MA, Schexnayder J, Patrician PA, Sales A. Implementation Science: New Approaches to Integrating Quality and Safety Education for Nurses Competencies in Nursing Education. Nurse Educ. 2017;42(5S Suppl 1):S12-s7.

18. Díaz-Barriga Arceo F. Los profesores ante las innovaciones curriculares. 2010. 2010;1(1)

19. Fernández Fernández IB, Guisasola Aranzabal G, Garmendia Mujika M, Alkorta Idiakez I, Madinabeitia Ezkurra A. ¿Puede la formación tener efectos globales en la universidad? Desarrollo docentes, metodologías activas y currículum híbrido. 2014;36(3):387-400.

20. Champin D. [Competency-based assessment in medical education]. Rev Peru Med Exp Salud Pública. 2014;31(3):566-71.

21. Garanhani ML, Vannuchi MTO, Pinto AC, Simões TR, Guariente MHDdM. Integrated Nursing Curriculum in Brazil: A 13-Year Experience. Creative Education. 2013;04(12):66.

22. Schoneman D, Simandl G, Hansen JM, Garrett S. Competency-based project to review community/public health curricula. Public Health Nurs. 2014;31(4):37383. 